\title{
The role of energy losses in photosynthetic light harvesting
}

\author{
T P J Krüger ${ }^{1}$ and R van Grondelle $e^{1,2}$ \\ ${ }^{1}$ Department of Physics, University of Pretoria, Private bag X20, Hatfield 0028, \\ South Africa. \\ ${ }^{2}$ Department of Physics and Astronomy, VU University Amsterdam, De \\ Boelelaan 1081, 1081 HV Amsterdam, the Netherlands. \\ E-mail: tjaart.kruger@up.ac.za
}

\begin{abstract}
Photosynthesis operates at the bottom of the food chain to convert the energy of light into carbohydrates at a remarkable global rate of about $130 \mathrm{TW}$. Nonetheless, the overall photosynthetic process has a conversion efficiency of a few percent at best, significantly less than bottom-up photovoltaic cells. The primary photosynthetic steps, consisting of light harvesting and charge separation, are often presented as having near-unity quantum efficiency but this holds only true under ideal conditions. In this Review, we discuss the importance of energy loss processes to establish robustness in photosynthetic light harvesting. Thermal energy dissipation of light-harvesting complexes in different environments is investigated and the relationships and contrasts between concentration quenching of high pigment concentrations, photoprotection (non-photochemical quenching), quenching due to protein aggregation, and fluorescence blinking are discussed. The role of charge-transfer states in light harvesting and energy dissipation is highlighted and the importance of controlled protein structural disorder to switch the light-harvesting antennae between effective light harvesters and efficient energy quenchers, is underscored. The main light-harvesting complex of plants, LHCII, is used as prime example.
\end{abstract}

Keywords: solar energy, light harvesting, photosynthesis, non-photochemical quenching, fluorescence blinking, single-molecule spectroscopy, charge transfer

\section{Introduction}

When exposed to different types of stress conditions, such as too high or too low temperatures, too little water, or too much absorbed light, the photosynthetic apparatus of plants, algae and cyanobacteria thermally dissipates a considerable fraction of the absorbed solar energy. Although this excessive amount of energy loss may be considered a major problem for bio-inspired solar technologies, it is important to view the 'problem' in a broader context and recognize the large variety of notable design principles. Obviously, photosynthetic organisms are more concerned about survival (i.e., fitness) than high biomass production (i.e., growth). For this reason, a vital property of the photosynthetic apparatus is functional robustness despite constantly fluctuating environments (i.e., 
disorder) on all levels of organization. Solar technologies based on low-cost molecular materials, such as polymers, organic semiconductors, and nanoparticles, face similar challenges of fluctuating environments and phototoxicity and may benefit greatly when steered by the design principles on which natural photosynthesis is operating.

Before highlighting some important functional aspects of thermal energy dissipation during the primary photosynthetic steps, the composition, characteristics and context of the photosynthetic lightharvesting apparatus will be introduced.

The basic light-harvesting apparatus of photosynthetic organisms consists of a reaction center (RC), the site where photochemistry takes place, surrounded by a network of light-harvesting antennae, forming a unit known as the photosystem (PS). This design ensures a large effective absorption cross section for each RC, thereby concentrating the incident excitations and providing an optimal photochemical turnover rate. Most photosynthetic organisms have two photosystems, called PSI and PSII. The RC of PSII is responsible for splitting water on the luminal side of the membrane and, using the energy of absorbed photons, it transfers the resulting electrons to a plastoquinone molecule. After a series of redox reactions, additional protons are enzymatically transferred through the membrane to the luminal side and electrons are transferred to the PSI RC. In summary, excitations are converted into a transmembrane proton and electron gradient, which is later used in the formation of ATP and $\mathrm{NADPH}$ (in oxygenic organisms) or NADH (in anoxygenic organisms), respectively, all of which are energy-rich molecules.

A light-harvesting antenna consists of a collection of pigments held in fixed positions and orientations typically by a protein. The pigments not only interact with one another but also with the protein. Both types of interactions may significantly alter their spectroscopic and light-harvesting properties. The protein constitutes a highly heterogeneous dielectric environment, exposing each pigment (bound at a specific site) to a unique charge density, which tunes the pigments' transition energy or 'site energy'. This modification in turn changes the inter-pigment couplings. In addition, the protein is a highly dynamic structure, exhibiting glass-like disorder on timescales ranging from sub-ps to s. The fast, collective, nuclear vibrational modes of the protein and pigments, known as phonons and often referred to as 'dynamic disorder', interact with the electronic excited states of the pigments, resulting in homogeneous broadening and energy shifts due to reorganization/solvation effects. The relatively slow protein structural fluctuations, known as 'static disorder', have a greater amplitude than phonons and may alter the light-harvesting efficiency of the pigment-protein complex. Specifically, static disorder generally gives rise to time-dependent fluctuations of the pigment site energies, and strong pigment binding to a moving protein residue introduces time-dependent changes in the inter-pigment couplings.

Let us consider the main light-harvesting complex (LHC) of plants, LHCII, to discuss one type of important energy loss process during light harvesting. This antenna complex naturally assembles into a three-fold symmetric structure (i.e., trimer) of identical subunits (i.e., monomers), each containing 14 chlorophyll (Chl) and 4 carotenoid (Car) pigments [1, 2]. The large conjugated ring of Chl supplies the molecule with a large absorption cross section and rigid structure. Only a relatively small modification of the ring is necessary to introduce large shifts in the transition energies. For example, the only difference between $\mathrm{Chl} a$ and $\mathrm{Chl} b$ is the identity of the small side chain $R$ shown in figure 1(a), but this difference translates into a shift of $0.085 \mathrm{eV}(30 \mathrm{~nm})$ of the lowest electronic transition, also known as $Q_{y}$ (figure 1(b)). The spectral tuning produced by the LHCII protein further increases the absorption spectral window of LHCII, creating an energy manifold (figure 1(c)). This natural gradient allows an excitation to rapidly relax to the site within the complex with lowest energy. The 
complexes are designed such that the lowest energy site is often located at the periphery, neighbouring other antenna complexes or the RC, thereby creating the important property of unidirectional excitation energy transfer. Energy relaxation along a gradient is obviously achieved by dissipating the amount of energy equal to the difference between the incident photon energy and the lowest site energy. But this partial energy dissipation considerably decreases the time for an excitation to reach the RC. Establishing unidirectional energy flow is therefore critically more important than an attempt to minimize energy loss. More generally, a non-equilibrium state, such as an excitation in a light-harvesting device, performs useful work when stabilized behind a barrier caused by heat dissipation [3].
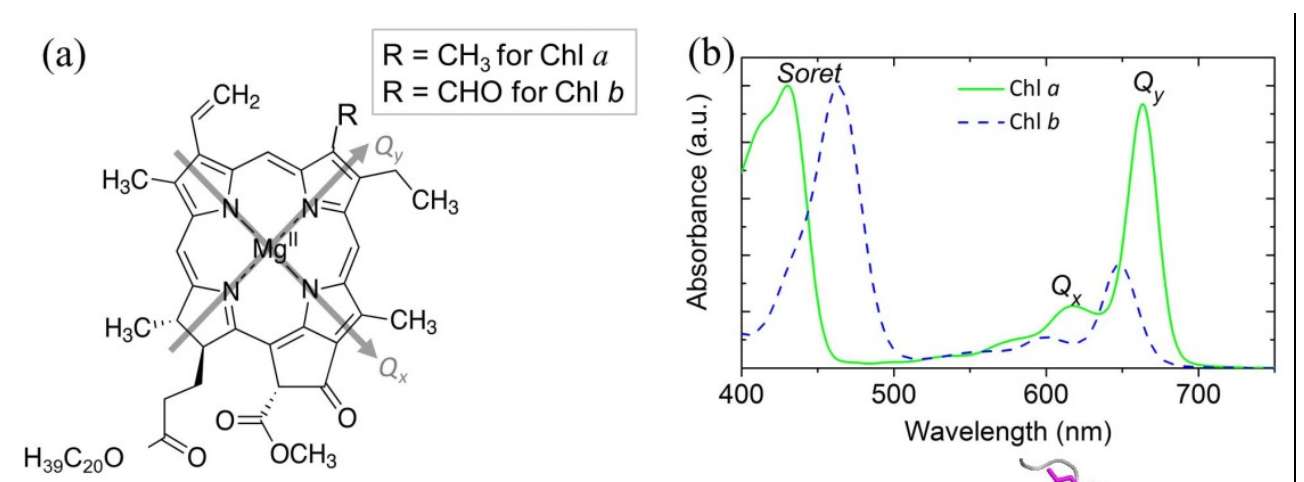

(c)
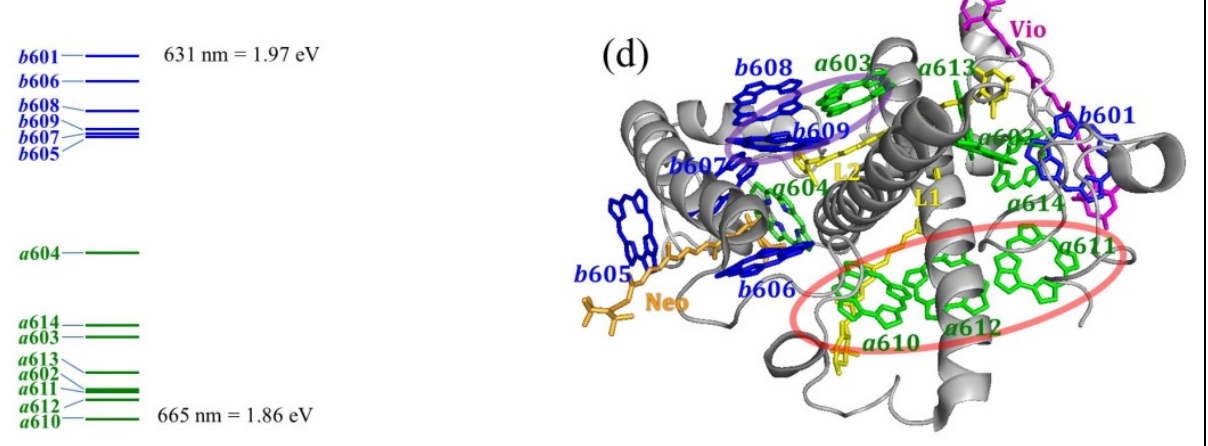

Figure 1. (a) Chemical structure of Chl $a$ and $b$. In gray are shown the directions of the $Q_{x}$ and $Q_{y}$ transition dipole moments. (b) Room-temperature bulk absorption spectrum of Chl $a$ (green solid line) and Chl $b$ (blue dashed line) in ethanol, normalized to the Soret peak maxima. (c) Site energy manifold of the Chls in a monomeric subunit of LHCII according to [4]. The values of the highest and lowest energy levels are shown. (d) Molecular structure of a monomeric subunit of LHCII at a stromal view, i.e., from the top of the thylakoid membrane. Data is based on the X-ray crystallographic model of [2] at a resolution of $2.72 \AA$ and the nomenclature used in [2] is indicated for all Chls and Cars. Chls $a$ and $b$ are depicted in green and blue, respectively, and only the chlorin rings are shown. The protein is displayed as four interconnected gray ribbons, and the four Cars are indicated by L1, L2, Neo, and Vio. Two Chl clusters that are discussed in the text are encircled, viz., 603-609 and 610611-612.

Another important function of energy loss is in the context of photoprotection. It is noteworthy that a constant energy throughput is maintained at the photochemical RC despite the frequent occurrence of solar intensity fluctuations of several orders of magnitude incident on, e.g., a leaf during the day [5]. The constant energy throughput, which is synchronized with the RC's turnover rate, is realized by the PSs constantly adapting to the incident light intensity, thereby limiting the formation of harmful 
photoproducts, such as reactive oxygen species formed during high excitation rates [6]. Rapid changes in the incident photon flux are accounted for by small structural changes of one or more of the lightharvesting proteins to open up energy dissipation pathways [7]. Slower intensity fluctuations are commonly dealt with by varying the antenna size, i.e., changing the effective absorption cross section for each PS, a phenomenon known as state transitions. This remarkable regulated behaviour leads to a strong reduction in the fluorescence that can be observed from plants and is therefore referred to as non-photochemical quenching (NPQ) of Chl $a$ fluorescence.

The two above-mentioned examples indicate two functionally vital types of (partial) excitation energy loss in the LHCs of oxygenic organisms. In this Review, we will focus on the unique property of critically high pigment densities in most photosynthetic LHCs and how this not only leads to delocalized excitation states with unique properties but also indispensably results in energy losses. We focus mainly on LHCII and investigate the molecular details of energy dissipation phenomena observed under various conditions, specifically upon protein aggregation and when the complexes are illuminated and detected individually. The possible molecular mechanisms of these processes are compared with NPQ and the phenomenon of concentration quenching of high Chl densities. We illustrate how protein static disorder can be revealed using single-molecule spectroscopy approaches and how this property is regulated functionally by photosynthetic organisms to equip them with environmentally controlled pigment-protein switches. In summary, the high pigment densities in photosynthetic LHCs are an ingenious design principle that provides the organisms with a robust and adaptable light-harvesting capacity.

\section{Photosynthetic excitons}

A remarkable property of most photosynthetic LHCs is their exceptionally high pigment density. For example, the Chl concentration in LHCII is $\sim 0.25 \mathrm{M}$ [2] (although concentrations of $>0.33 \mathrm{M}$ are often reported in literature - e.g. [8]), which is equivalent to average nearest-neighbour Chl-Chl separations of $<11 \AA$ [2]. Center-to-center separations as short as $8 \AA$ are found for some Chl pairs in LHCII [2]. This design not only ensures an optimal effective absorption cross-section but the resulting strong inter-pigment couplings (up to $110 \mathrm{~cm}^{-1}$ ) [4] also give rise to new physical states, known as Frenkel excitons, whereby an excitation is delocalized over a number of strongly interacting pigments and hence coherently shared amongst those pigments [9]. Excitons introduce important, new functions. Energy relaxation amongst exciton states not only occurs faster than resonance energy transfer between individual pigments but excitons also speed up energy relaxation along an energy gradient due to the reduced number of steps required [10,11]. Even in the case of isoenergetic energy migration between LHCs and the RC, the number of pathways that need to be explored is significantly decreased in the exciton picture than when considering excitations localized to individual pigment sites. As a consequence, energy migration times are significantly shorter and the quantum efficiency is enhanced. PSI, containing a network of $\sim 200$ Chls, transfers excitation energy within 20-30 ps to the RC [12], whereas the larger PSII-LHCII supercomplex requires only a slightly longer time (30-50 ps) to do the same [13-15]. These time scales are 100 times faster than the intrinsic decay lifetime of a Chl electronic excited state of $\sim 4$ ns in a protein environment such as LHCII, lending these complexes near-unity quantum efficiencies for trapping the absorbed solar photon.

Due to their extended nature, excitons are much less affected by excitation traps due to single site defects in the antenna network than are localized excitations. This general way of avoiding the adverse effects of disorder is significantly more economic than creating a defect-free, crystalline 
material. However, disorder tends to localize excitations due to exciton-phonon coupling, which, in photosynthetic LHCs, is of the same order of magnitude as typical inter-pigment coupling strengths. The result is a reasonably short exciton delocalization length of typically 2-4 pigments [16], which is sufficiently small to provide the complexes with the necessary functional dynamicity, for example to probe different possible energy transfer pathways and switch between light-harvesting and photoprotective states.

Two excitonically coupled clusters in LHCII that will be considered in this Review are shown in figure 1(d). We will show that the cluster of three Chls near lutein L1 is strongly involved with NPQ, while the cluster of two Chls near lutein L2 gives rise to charge-transfer states, which significantly lower the exciton energies.

\section{Concentration quenching}

For pigment densities as high as those occurring in most photosynthetic antenna complexes it is not trivial to have efficient energy transfer without significant losses. It was demonstrated over 40 years ago that when free Chls are solubilized at a concentration of $>0.1 \mathrm{M}$, effectively all excitations are rapidly quenched due to a process known as concentration quenching or self-quenching [17]. In contrast, photosynthetic LHCs circumvent this type of quenching by a precise arrangement of their pigments in the protein matrix. One important question, which is rarely posed, though, is whether concentration quenching is really completely prevented in the natural protein host, or, more generally, whether it may actually be used to play a photoprotective role. To address this question, we will first investigate the possible molecular mechanisms of concentration quenching and NPQ and thereafter consider the role of protein disorder.

In the 1970s, Sir George Porter studied the phenomenon of concentration quenching extensively. By means of careful experiments on Chls and other pigment molecules in various environments he successfully ruled out a number of possible molecular mechanisms. First, due to the particular quadratic dependence of the fluorescence yield on the pigment concentration, intersystem crossing, multi-excitation annihilation and collisional quenching could be excluded as quenchers [18]. Second, impurities as energy traps are highly unlikely due to the independence of the quenching rates on different solutes and on the addition of impurities to the solution [18, 19]. Third, Chl aggregation generally leads to spectral changes, which were not observed in the concentration quenching studies of Chl. Furthermore, studies of Chls in lipid solvents showed no deviation from Lambert-Beer's law [18], also ruling out Chl aggregation as a likely explanation. Nonetheless, Katz and co-workers promoted the idea of Chl aggregation being the source of fluorescence quenching [20]. Curiously, at high concentrations, most other pigment molecules tend to aggregate, which is an important consideration for technologies such as dye-sensitized solar cells. Chls have also been observed to aggregate in certain solutions (e.g., [20-22]). However, in organic solvents with a dielectric constant similar to that of proteins, Chls do not aggregate, although pheophytins, which are Chl molecules without the central $\mathrm{Mg}$ ion, do aggregate in organic solvents [18, 23], suggesting an important role of the $\mathrm{Mg}$ ion to prevent Chl aggregation.

Porter attributed concentration quenching to energy transfer to "statistical pairs", which are nonradiative excited-state dimers separated less than a critical distance [18]. At this critical distance an energy trap is formed. In their view, in such a concentrated Chl suspension, an excitation will diffuse amongst Chls by means of Förster resonance energy transfer until it reaches such a statistical pair, 
whereupon the excitation will be quenched. The pairs are called "statistical" because (a) the critical distance, being smaller than the average intermolecular distance, has a certain statistical probability of being reached between neighbouring pigments, and (b) an inter-pigment separation less than the critical distance occurs only for a brief moment, in contrast to permanent Chl aggregates. Based on the best fit of their data, they determined a critical distance of $10 \AA$ for Chls.

Let us consider the average Chl concentration of $0.25 \mathrm{M}$ in LHCII. Solubilized at this concentration gives an average Chl-Chl center-to-center distance of $10.4 \AA$. When including sizeable variations around this distance due to kinetic energy and the accompanying statistical speed distribution, a large fraction of Chls will be within $10 \AA$ of their nearest neighbour at any given time and most excitations will be quenched according to the model and observations of Porter and co-workers.

Although Porter and co-workers eliminated a number of molecular mechanisms, they did not provide details about the nature of the energy trap within a statistical pair of Chls. Let us investigate some possibilities. The critical distance of $10 \AA$ is much smaller than the van der Waals radius of the Chl ring of $\sim 14 \AA$ and $\sim 16 \AA$ along the $Q_{x}$ and $Q_{y}$ molecular axes, respectively. The probability of reaching the critical distance is therefore highest for a co-facial arrangement of the rings of a Chl dimer. Such arrangement corresponds to strong excitonic couplings. Furthermore, at this distance, there is room for only a couple of (small) solvent molecules between the Chls and therefore little screening of the strong intermolecular coupling takes place. Considering that the average nearestneighbour distance of $\sim 10.4 \AA$ in LHCII is surprisingly near the critical distance and that the kinetic energy of the Chls leads to sizeable variations around this value, Chl-Chl separations significantly less $10 \AA$ may be expected. However, a strong excitonically coupled Chl dimer does not constitute a nonradiative energy trap but rather modifies the transition dipole moments. For example, strongly coupled Chls in a co-facial arrangement leads to a blue-shifted spectrum, since the red-shifted exciton has only a weak oscillator strength $[9,24]$.

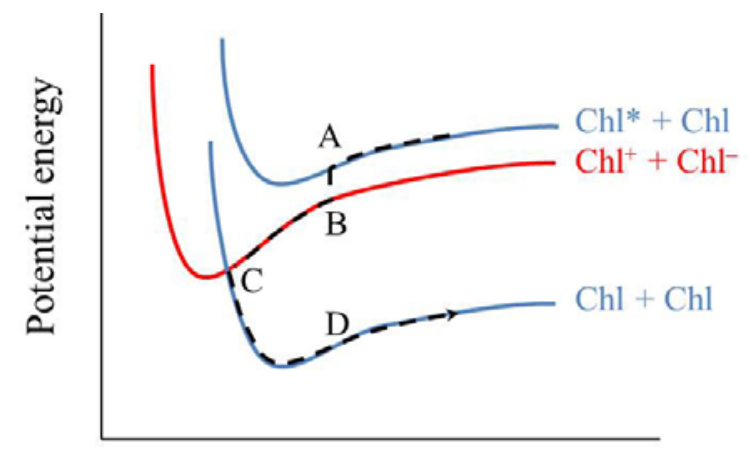

Chl-Chl separation

Figure 2. Energy surfaces of different states of a Chl dimer, explaining the molecular mechanism of concentration quenching proposed by [25]. Initially, one of the Chls in the dimer is excited (Chl*, top) and transfers an electron to the other $\mathrm{Chl}(\mathrm{A} \rightarrow \mathrm{B})$. The resulting ion pair attracts each other electrostatically, progressively decreasing the energy as the inter-nuclear distance decreases $(B \rightarrow C)$, and collapses non-radiatively to the ground state $(C \rightarrow D)$.

Considering that at $10 \AA$, Dexter energy transfer and charge transfer (CT) become likely and transient changes in polarizability and dipole start to occur, it seems likely that partial or complete electron transfer from one $\mathrm{Chl}$ to another may be involved with the underlying mechanism. This view was 
postulated by different groups during the early, extensive studies of concentration quenching [25-27], partly on the ground that it is energetically feasible (figure 2). Later theoretical studies have indicated that in photosynthetic LHCs, the energy of CT states is generally close to some of the exciton states $[28,29])$ and it is very likely that the same applies to strongly coupled free Chls. The electrostatic attraction resulting from the CT state, or ion pair, will draw the Chls even closer together and prolong their interaction. After rearrangement of the solvent dipoles within a few ps, recombination of the ion pair takes place and an adiabatic transition to the ground state is made [25]. The latter transition occurs rapidly if the ground state energy surface crosses near the minimum of the ion pair's energy surface (figure 2). Such rapid collapse of the ion pair through internal conversion may explain the lack of experimental evidence for CT state formation or radical-ion intermediates. However, in the '70s, laser spectroscopy was limited to the ps timescale and faster processes could not be observed. In a quest to identify the molecular mechanisms underlying Chl concentration quenching it is advisable to resume the investigation using modern-day state-of-the art visible-NIR fs spectroscopy techniques.

\section{Non-photochemical quenching (NPQ)}

In contrast to the stochastic nature of concentration quenching, NPQ is a finely regulated process that depends on the collective behaviour of several proteins featuring over timescales ranging from seconds to hours. The fastest component, commonly referred to as $\mathrm{qE}$, takes place in PSII and it is widely accepted that a prominent part of it takes place in LHCII. NPQ responds as a feedback mechanism to the transmembrane $\mathrm{pH}$ gradient. Such a gradient is formed when the energy delivered to the PSII RC is faster than the rate of electron transport from PSII to PSI, resulting in a decrease of the luminal $\mathrm{pH}$. As a result, the LHCs in PSII are protonated [30], one specific Car bound to LHCII, violaxanthin, is enzymatically de-epoxidized into zeaxanthin [31], and the PSII subunit known as PsbS is protonated and becomes involved with qE in a way not yet entirely clear [32]. These $\mathrm{pH}-$ induced changes are accompanied by a characteristic Raman signal in LHCII [7], indicating a small but specific protein conformational change in this complex. The consensus is that protein conformational changes associated to $\mathrm{qE}$ redistribute the energy levels of particular pigments in the complex, opening energy dissipating channels that would trap the energy transferred amongst the Chls.

Different possible molecular mechanisms have been proposed, all of which can be reduced to some particular strong interaction within a pigment dimer, either Chl-Chl or Chl-Car. Two types of energy traps have gained prominence: A low-lying Car $\mathrm{S}_{1}$ state [33, 34] and a CT state formed in a Chl-Chl dimer [35, 36] or in a Chl-Car dimer [37, 38]. Evidence for the first type of trap was given in the landmark paper of [34] wherein the results from time-resolved absorption experiments on strongly quenched LHCII aggregates showed incoherent energy transfer from a low lying Chl exciton state to a Car $S_{1}$ state, followed by rapid internal conversion. The carotenoid was identified as the lutein L1 in the atomic structure [2] of LHCII, indicating that the closely coupled exciton state is associated with the Chl a610-a611-a612 cluster. This model was later corroborated in theoretical studies, which showed that the particular arrangement of L1 combined with its close contact with the Chl $a 610-a 611$ a612 cluster allowed small configuration changes of L1 to result in fast energy transfer from the Chl cluster to the short-living $\mathrm{S}_{1}$ state of L1 $[39,40]$. Due to the close proximity of L1 to Chls a610a611-a612, strong coupling between all these pigments is expected and as a result also coherent mixing between the L1 $\mathrm{S}_{1}$ state and the lowest exciton states of the Chl cluster. The latter explanation was assumed in [41, 42], and later confirmed by two-photon absorption experiments [43] and quantum-chemical calculations [44]. 
The hypotheses favouring the second type of energy trap - involvement of a CT state - are currently only based on peripheral evidence. Holt and co-workers observed a new NIR signal using femtosecond transient absorption spectroscopy and showed that this signal coincides with the groundstate absorption spectra of $\beta$-carotene and spheroidene radical cations [37]; however, these Cars are not present in the LHCs of PSII, and, more importantly, no corresponding change in the Chl $Q_{y}$ region was reported. Holzwarth and co-workers based their interpretation on the characteristic red-shifted emission that is known to accompany NPQ [35, 36]. Indeed, the low-energy states characteristic of several different types of LHCs have been effectively explained using models that incorporate exciton/CT mixed states [45-50]. The generally strong coupling of CT states to phonons gives rise to sizeable displacements along the nuclear coordinates as compared to the excited states. Exciton-CT mixing therefore results in a significant redistribution of the dipole moments and the associated reorganization energy gives rise to a large Stokes shift. The exciton/CT mixed state also borrows transition dipole strength from the exciton state, whereby it becomes weakly emissive, and it borrows some of the static dipole from the CT state, whereby the Stark signal increases. Stark fluorescence spectroscopy confirmed the presence of CT states in strongly quenched LHCII aggregates [51]. In fact, this study showed evidence for two distinct CT signals, which were attributed to a Chl-Chl and Chl-Car dimer, respectively, both of which were assumed to be responsible for strong energy dissipation. However, in all these models causality is assumed, i.e., the simultaneous appearance of two phenomena implies that one is responsible for the other. It was recently shown that the temperature-dependent time-resolved fluorescence spectra of strongly quenched LHCII aggregates can only be adequately interpreted when using a three-state model wherein quenching and red emission correspond to different states [52]. The latter study confirmed what was earlier concluded from single-molecule spectroscopy experiments [53], indicating that the emissive exciton/CT mixed states are not the main quenchers but just happen to be present during $\mathrm{qE}$.

\section{Protein Aggregation}

It is important to note that most of the above-mentioned studies on possible molecular mechanisms of $\mathrm{qE}$ were not performed on LHCII in its native environment or even on free, isolated complexes, but LHCII aggregates were used, i.e., large conglomerates of closely spaced complexes. Such aggregates are generally formed by solubilization of isolated LHCII complexes in a solution resembling the natural environment, followed by partial removal of the detergent so that the protein-detergent phase equilibrium is shifted towards larger protein units. This procedure generally leads to the formation of large three-dimensional aggregate structures. However, a molecular study of NPQ is hardly possible in vivo, firstly because the effective fluorescence yield is very small (at most a couple of percent) due to the fast and efficient trapping by the RC, and, secondly, PSI and PSII are competing for the same photons and display strongly overlapping spectroscopic responses and kinetics. Nonetheless, LHCII aggregates display numerous spectroscopic features that are also observed for NPQ in vivo, such as emission around $700 \mathrm{~nm}$ [35, 54-56]. The same spectroscopic features were also observed from isolated, quenched LHCII complexes in a gel matrix and in a crystalline arrangement where only weak inter-complex interactions were present and no protein aggregation occurred [7,57]. It was concluded that the accompanying conformational changes are intrinsic features of each LHCII trimer and not artificially created by aggregation. Furthermore, freeze-fracture electron micrographs revealed clustering patterns of LHCII complexes during NPQ, providing evidence that some aggregation occurs during NPQ in vivo [58]. 


\section{Fluorescence blinking}

Considering the high pigment density in LHCII it is not surprising that perturbations may quite easily result in quenching. In fact, the combination of exceptionally dense pigment packing and the large amount of protein disorder at physiological temperatures appears to be a perfect recipe for periodic quenching of the excitation energy. This is exactly what is observed when the fluorescence of individual LHCII complexes is monitored (figure 3(a)). Due to the stochastic nature of the protein dynamics, disorder-related fluorescence variations are averaged out when even a small number of non-interacting complexes are observed simultaneously. However, single-molecule spectroscopy (SMS) enables the study of fluorescence spectral changes on a time scale where physiologically relevant conformational changes take place.
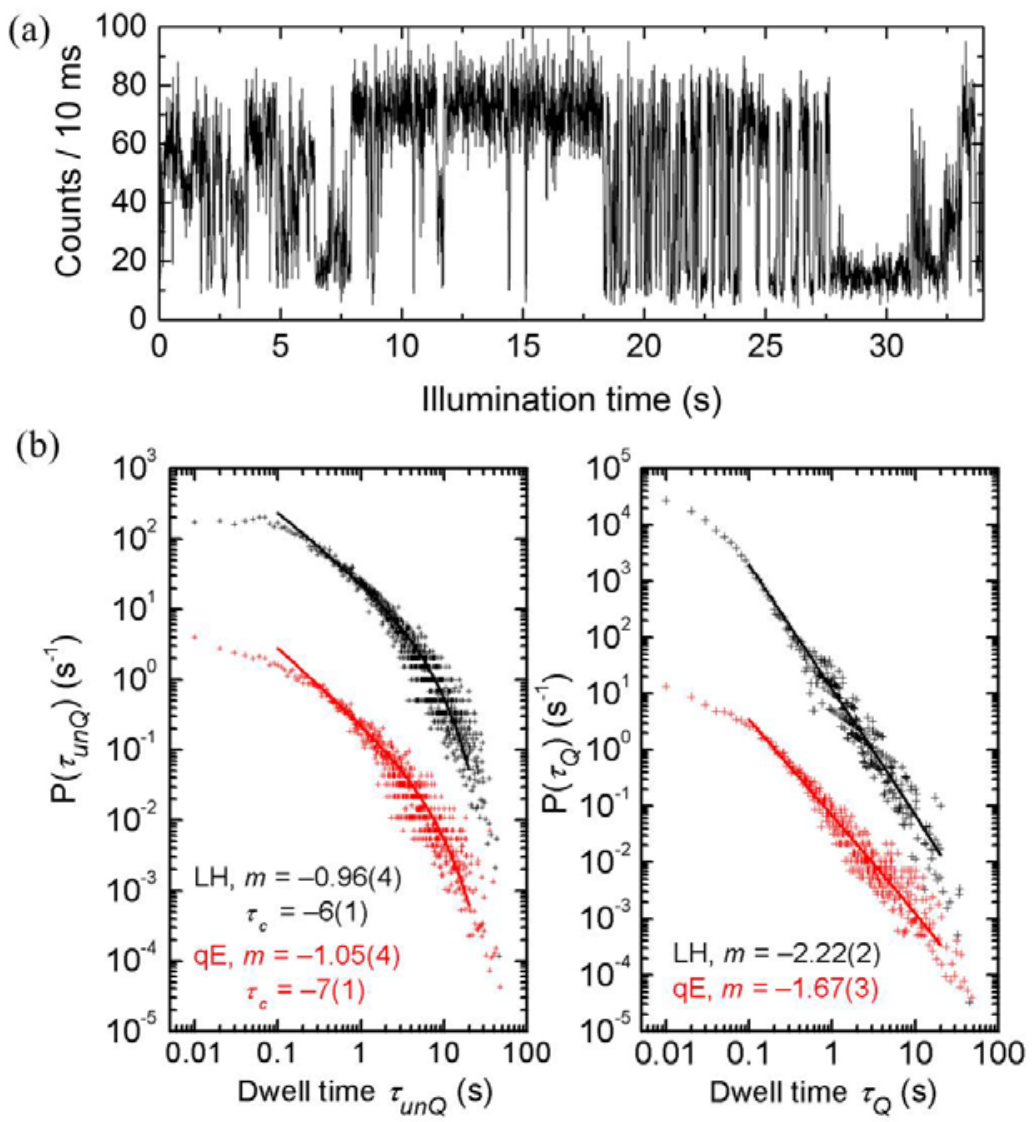

Figure 3. (a) Example of fluorescence blinking of a single LHCII complex. (b) Weighted probability distribution of the dwell time in an "off" state $\left(\tau_{Q}\right)$ and in an "on" state $\left(\tau_{\text {unQ }}\right)$ in an environment mimicking efficient light-harvesting ( $\mathrm{LH}$, black) or qE (red). The weighted probability density of a dwell time $\tau$ was defined as $P(\tau)=\Delta t_{a b}^{-1} N(\tau) / N_{\text {tot }}$, using the weighting factor $\Delta t_{a b}=(a+b) / 2$, where $a$ and $b$ are the time differences between $\tau$ and the next longest and next shortest dwell times with nonzero probability, respectively. $N(\tau)$ denotes the number of intensity levels with dwell time $\tau$ and $N_{\text {tot }}$ is the total number of intensity levels. Least-squares fits (lines), together with the fitting results, are shown, using equations $P(\tau) \propto \tau^{m}$ for quenched intensities (i.e., "off" states) and $P(\tau) \propto \tau^{m} e^{-\tau / \tau_{c}}$ for unquenched intensities (i.e., “on” states). Here, $m$ denotes the power-law slope 
and $\tau_{C}$ the characteristic time after which an exponential behaviour dominates the power-law behaviour. Standard errors of fits are indicated in brackets. Black data points are offset for clarity.

Figure 3(a) shows the large, rapid, reversible intensity changes that are typically observed when a single LHCII complex is continuously illuminated with a constant photon flux. This phenomenon is, however, not unique to LHCII or even pigment-protein complexes in general, but almost any type of nanoscale fluorescent object is known to exhibit such behaviour, generally known as fluorescence intermittency or blinking. For semiconductor quantum dots and single dye molecules, the dominant blinking mechanism involves ionization. For quantum dots, such ionization results from a photoassisted Auger process [59,60], while for pigment molecules, long-living, dark radical states are typically populated via triplet states [61]. Considering the large collection of pigments in LHCII, one might similarly expect radical states to form stochastically on any one of the pigments, which would trap all subsequent excitations in the complex. However, such a hypothesis lacks experimental evidence. Radicals have as yet only been reported from carotenoids in isolated LHCII after very specific double excitation of the carotenoids using resonant two-colour, two-photon ionization spectroscopy [62]. Furthermore, carotenoid radical cations may form in one of the photosystems during NPQ [37], but it is strongly debated whether those cations originate from LHCII. In any case, the sub-nanosecond carotenoid radical lifetime is much too short to be relevant for the ms $-\mathrm{s}$ dynamics of fluorescence blinking.

In a recent SMS study on LHCs from cyanobacteria it was found that the intensity fluctuations decreased as the protein rigidity was increased by using cross-linkers, providing strong evidence that protein static disorder underlies fluorescence blinking in these complexes [63]. It is highly likely that protein conformational dynamics similarly governs blinking in LHCII, explaining the quasistability of the fluorescence intensity on timescales of $\mathrm{ms}-\mathrm{s}$.

One important question in this context is whether the blinking-related energy traps, which reflect intrinsically available energy traps, are also used for a functional purpose in the native environment. A negative answer would imply that unique protein conformations are created during $\mathrm{qE}$, which would open very specific energy dissipating pathways. This question was addressed by investigating fluorescence blinking from individual LHCII complexes in the context of NPQ and comparing the results with the highly homologous monomeric minor complexes that are neighbouring LHCII in PSII [53, 64]. To this end, different conditions known to be involved with $\mathrm{qE}$ in vivo were mimicked. Specifically, an acidic environment was used, with a $\mathrm{pH}$ similar to that found in the lumen during strong $\mathrm{qE}$ conditions; the concentration of the detergent, which mimics the membrane within which the complexes are naturally embedded, was drastically decreased; and violaxanthin was replaced with zeaxanthin. For LHCII, the combination of these three environmental changes gave rise to a significantly longer average dwell time in quenched states (figures 3 and 4). In particular, figure 3(b) shows the probability density distribution of "on" and "off" dwell times, assuming a two-state model, where "on" and "off” refer to strongly and weakly emitting states, respectively. "Off” states followed largely a powerlaw dependence, with a strongly decreasing slope under qE-mimicking conditions, while the "on" states showed a powerlaw behaviour (with an exponential tail at longer dwell times), and the powerlaw slope somewhat increased when $\mathrm{qE}$ was mimicked. Including also the significant contribution of intermediate intensity levels, commonly observed from multi-pigment systems, the intensity probability distributions are shown in figure 4(a), and the shift to quenched states is evident in the qE-mimicking environment. When including the so-called dim fraction - intensity traces of 
complexes whose "on" states were related to a significantly lower intensity as those shown in figure 4(a) - promotion of quenched states became even more prominent (figure 4(c)). For the minor peripheral antenna complexes of PSII, an entirely different environmental response to qE-related conditions was found. When excluding the dim fraction, the equilibrium between quenched and unquenched states showed the opposite response as for LHCII (figure 4(b)); when including the dim fraction, the intensity distribution largely broadened and the population-average intensity was found to be independent of the environmental conditions (figure 4(d)).
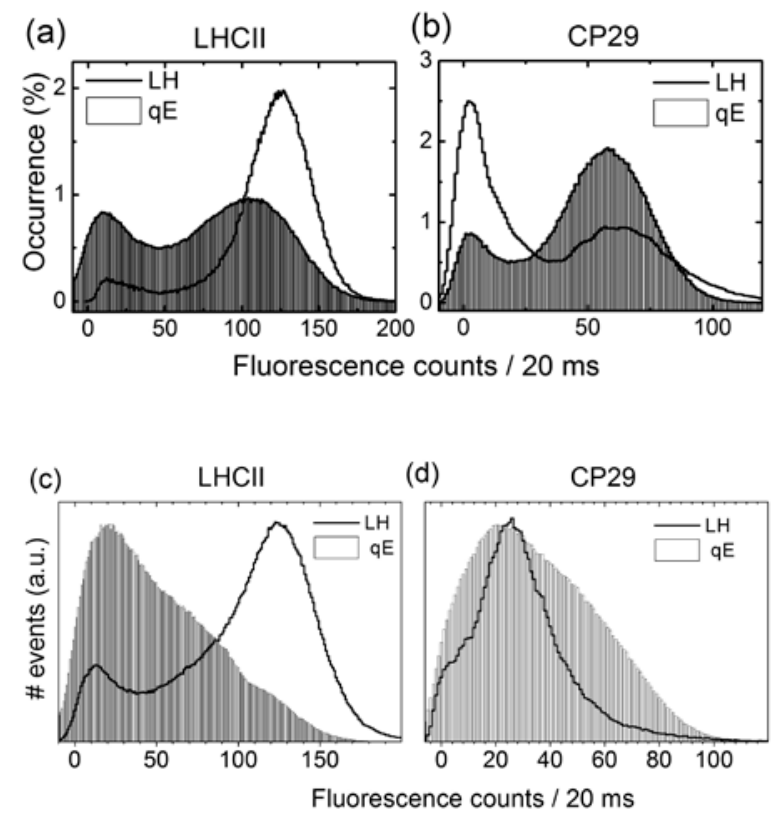

Figure 4. Intensity distributions of an ensemble of individually measured (a) LHCII trimers and (b) CP29, representative of the minor antenna complexes of PSII. Distributions are displayed for an environment mimicking low light (light harvesting, LH, open histograms) and high light (quenching, $\mathrm{qE}$, filled histograms) conditions, including in the analyses only complexes that displayed a high maximum intensity [53]. (c) and (d) show intensity distributions under the same conditions, but where the dim fraction of complexes were included in the analyses [64].

The results in figures 3 and 4 strongly suggest a direct relationship between fluorescence blinking and $\mathrm{qE}$ that is specific for LHCII, i.e., the molecular mechanism underlying fluorescence blinking in LHCII plays an important role in NPQ. In other words, an intrinsically available thermal energy dissipation state is used for the purpose of photoprotection and the probability of accessing this state is strongly enhanced under NPQ conditions.

A two-state model was developed to describe fluorescence blinking in LHCII, based on structural diffusion of the protein surrounding the Chls a610-611-612 domain [65, 66]. In this model, the "on" and "off" states are each represented by a harmonic potential energy surface and switching between the two states is determined by a random walk of the protein structure, which modulates the probability of excitation energy transfer from the terminal emitter Chl cluster to the $S_{1}$ state of the nearby lutein L1, a qE-related energy trap [34]. Using this model, most of the fluorescence blinking statistics could be reproduced [65-67] and pH-dependent changes in the dwell time probability distributions (figure 3b) could successfully be accounted for by considering $\mathrm{pH}$-dependent model parameters, thus providing qualitative support for a connection between fluorescence blinking and $\mathrm{qE}$. 
Recent molecular dynamics simulations showed evidence of significant changes in the relative positions and orientations of L1 and Chls $a 611$ and 612 [68], which beautifully fits the idea of protein static disorder underlying fluorescence blinking.

Curiously, the largest equilibrium shift to quenched states was observed when reducing the detergent concentration of surface-bound, well-separated LHCII complexes [53]. Such an environment partially mimics the effect of aggregation of single LHCII complexes due to the likely small solvent-exposed unfolding of the protein at its surface [69]. The strong enhancement of thermal energy dissipation could be explained by the propagation of such surface changes to physiologically relevant structural changes in the protein core to increase, for example, Car-Chl coupling in the context of qE.

\section{Comparison of different quenching environments}

We have indicated a connection between fluorescence blinking and NPQ for LHCII, between NPQ and LHCII aggregation, and also some resemblance between fluorescence blinking and aggregation. Although the relationships suggest some commonality in the molecular mechanisms underlying the different phenomena due to their response to qE-related conditions, the number of possible mechanisms may vary. For example, additional energy traps are very likely formed in protein aggregates, such as inter-subunit Chl-Chl quenching, binding of non-functional or denatured complexes (i.e., defects) that act as energy traps, additional protein conformational changes due to inter-complex forces, and some degree of unfolding of the protein surface. Considering the high pigment density in LHCII, subtle protein conformational changes should be sufficient to give rise to quenching. We need to distinguish between spatial and energetic modifications of the pigments. Due to the strong distance dependence of dipole-dipole interaction, the probability for energy trapping is more sensitive to spatial variations of the pigments. Slight compression of LHCII is therefore expected to increase the quenching probability. Indeed, hydrostatic pressure experiments on isolated LHCII complexes [70] revealed that energy dissipation and a small red shift of the fluorescence spectrum are directly correlated with the amount of external pressure exerted on the complexes. A similar mechanism may partially occur during protein aggregation.

Concerning fluorescence blinking, it has been shown for LHCII [71], bacterial LHCs [72, 73] and cyanobacterial LHCs [63] that the rate of intensity fluctuations increases as a function of the incident photon flux, favouring quenched states, suggesting that the underlying mechanism is strongly lightdriven. However, in the cyanobacterial LHC study, blinking was still observed at physiological light intensities [63]. In addition, there is strong evidence that fluorescence blinking in LHCII is not specific to a particular SMS environment. For example, blinking was observed when immobilizing the complexes to a glass substrate using non-specific electrostatic interactions [74] as well as when the motion of the complexes was counteracted in solution using electrokinetic forces [75]. Furthermore, when averaging the results of a large population of individually measured complexes, the photophysical properties, such as the fluorescence lifetime, triplet yield, and fluorescence lineshape, were the same as those obtained from bulk studies [53, 76]. It may be argued that static disorder is less prominent when the complexes are not isolated but part of a PS due to inter-complex interactions that may restrain some of the protein degrees of freedom. However, a recent SMS study on whole PSII-LHCII supercomplexes shows that fluorescence blinking occurs in the individual LHCs despite their interaction in the supercomplex [77]. The above-mentioned studies strongly suggest that the energy traps responsible for fluorescence blinking should also be present in LHCII in vivo. In a recent ultrafast multiphoton spectroscopy study on LHCII, a new dark state was discovered and proposed to 
be related to the mechanisms underlying fluorescence blinking as well as the strong quenching exhibited by LHCII aggregates [78].

Is there any relationship between $\mathrm{qE}$ and concentration quenching? For the high pigment densities in LHCII, inter-Chl distance is an important parameter and small changes may switch the complex between light-harvesting and energy-trap states. This is also the idea behind the "statistical pair" in Porter's model for Chl concentration quenching, which forms below a critical distance. There are two important differences between solubilized Chls and the pigment arrangement in LHCII, though. First, the random distribution and kinetics of Chls in solution strongly increase the probability of forming a statistical Chl pair as opposed to the precise arrangement and motional limitations brought about by the LHCII protein, which may be finely controlled to establish efficient light harvesting of quenching. Second, in Porter's model, only Chl pigments are present, whereas in LHCII, the presence of Cars adds a few important possibilities for excitation energy traps, in particular due to their dark, shortliving $S_{1}$ states. However, in all the above-mentioned quenching environments, CT states in Chl dimers appear to be important. In a recent study on bacterial LHCs it was shown that dark, transient states are accessed during equilibration along an energy gradient, which were attributed to CT states or polaron pairs in bacteriochlorophyll dimers [79]. It was concluded that the formation of CT states is unavoidable for the high pigment densities of photosynthetic LHCs and are often used to enhance unidirectional energy transfer. However, slight shifts in the energy levels of such CT states or the pigment arrangement may turn the CT states into effective energy traps. A recent SMS study on cyanobacterial LHCs has provided evidence for a relationship between long-living off states and strongly red-shifted emission states [63], suggesting a role of CT states in energy quenching for isolated LHCs containing no Cars. The evidence for prominent CT states as revealed by a Stark spectroscopy experiment on LHCII aggregates [51, 80] could suggest that the promotion of CT states is enhanced during aggregation. We conclude that the main mechanism underlying $\mathrm{qE}$ probably involves Chl-Car interactions, while quenching due to CT states, possibly from Chl dimers, may play a secondary role. It is likely that Nature uses all available energy loss mechanisms for a functional purpose, including that of concentration quenching, and does this by finely controlling the equilibrium between accessing and avoiding energy traps.

\section{Low-energy emission: control of shade absorption}

The static disorder of LHCII also gives rise to a significant amount of spectral diffusion (figure 5). To shed light on the possible origin of spectral changes, a disordered exciton model, based on modified Redfield theory [4], was used [81]. In this model, the static disorder was simulated by randomly varying each Chl site energy, assuming a Gaussian probability distribution around the ensembleaverage values, and thereafter calculating the resulting energy equilibration and steady-state fluorescence spectrum. A small fraction of realizations of the disorder (1-3\%, depending on the width of the disorder assumed in the model) gave rise to spectral shapes that deviate from the ensembleaveraged spectrum. These deviating spectral shapes had a maximum intensity that peaked within 10 $\mathrm{nm}$ of the ensemble spectral peak at $\sim 682 \mathrm{~nm}$ and corresponded well with the experimentally measured single-molecule spectra. Relatively small spectral variations can therefore be explained by site energies variations due to static protein disorder, which in turn determine the specific exciton states that contribute most to the fluorescence.

Under continuous illumination, a fraction of complexes ( 3-5\%) reversibly visit spectral states with a peak wavelength near $700 \mathrm{~nm}$ (figure 5(a)) [81, 82], while numerous spectra have been observed at 
even longer wavelengths, occasionally with shifts of more than $100 \mathrm{~nm}$ to the red [53]! These redshifted states cannot be explained by site-energy disorder but require the protein to switch into particular conformations to invoke special pigment interactions. Curiously, the fraction of complexes exhibiting emission near $700 \mathrm{~nm}$ was found to slightly increase in a qE-mimicking environment [82]. It therefore does not seem coincidental that emission around $700 \mathrm{~nm}$ is widely considered to be a signature of qE [35, 56]. Moreover, besides being promoted simultaneously by qE-conditions, no other relationship was found between red states and fluorescence blinking. For example, the fluorescence intensity also fluctuated when red emission was exhibited by the complexes, and the absolute intensity of the $680-\mathrm{nm}$ and 700 -nm states was comparable. It was therefore concluded that blinking and red emission originate from different sites in LHCII, corroborating the three-state model of [52]. The lack of causality between the two phenomena can be explained by the conformational state involved with $\mathrm{qE}$ also promoting changes in the site where the $700 \mathrm{~nm}$ emission originates [82]. Figure 6 shows how LHCII exists in a dynamic equilibrium between a small number of different quenched and spectral conformations and shows the most likely sites associated with the different spectroscopic states. In this model, the protein structural disorder is responsible for frequent switching between the different quasi-stable states, while the local environment in the L1 and L2 domains determines which of these states are favoured. As such, fine control of LHCII's intrinsic switching capability is established.
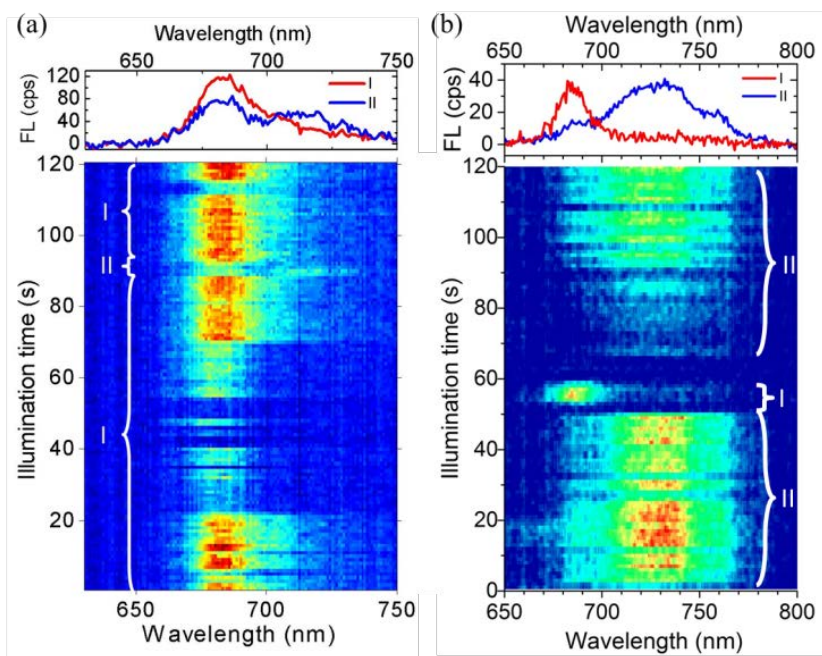

Figure 5. Representative example of time-resolved fluorescence spectra showing large reversible spectral shifts from (a) a single LHCII complex [81], and (b) a single Lhca1/4 dimeric complex on the periphery of PSI [83]. The Lhca1/4 dimer remarkably switched off its entire red spectral component. Fluorescence counts were binned into an integration time of $1 \mathrm{~s}$. Spectra on top are the time averages of the distinct spectral states indicated by I and II respectively. 


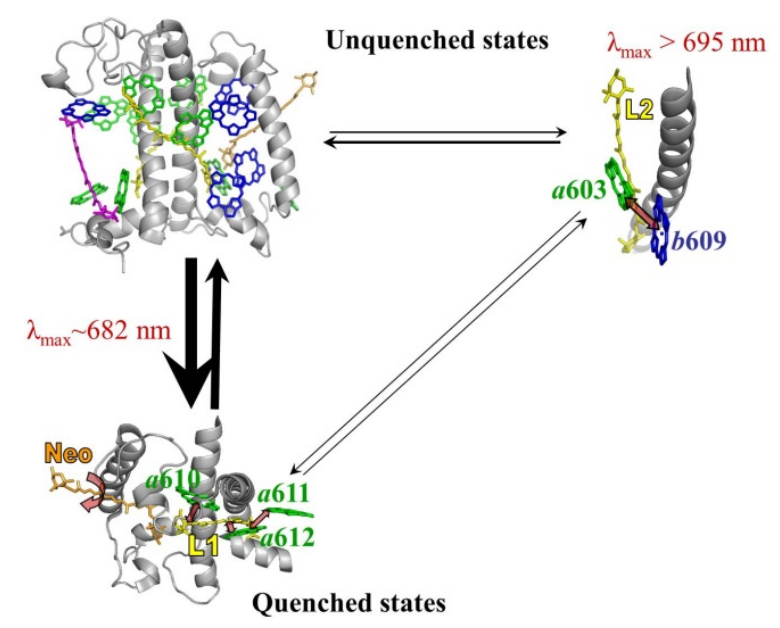

Figure 6. Three-state dynamic model for the emission from an LHCII subunit, showing the most likely sites of origin for different spectral features. Specifically, the LHCII monomeric structure [2] in an unquenched state exhibits emission at $\sim 682 \mathrm{~nm}$, red-shifted emission most likely originates from the formation of a CT state in a Chl dimer close to lutein L2 (top right) [81, 83], while fluorescence blinking and qE mostly originate from coupling between the terminal emitter Chl cluster and the lutein L1 (bottom left) [34]. The black arrows represent transitions between different states, with the thickness giving a qualitative indication of the frequency of occurrence. The straight, red arrows signify strong interactions; the curved arrow indicates a configurational twist of the carotenoid neoxanthin.

Although relaxation along an energy gradient in LHCs may be considered an energy loss process, it is remarkable that a large number of photosynthetic LHCs are known to enlarge their absorption bandwidth into the red due to the presence of so-called red pigments that absorb light at lower energies than utilized by the RC [12, 48]. A similar property is unlikely for semiconductors, which would mean that photons with energy smaller than the bandgap should be absorbed and utilized efficiently. Plant PSI contains two peripheral LHCs that have fluorescence spectral peaks beyond 720 $\mathrm{nm}$, despite their proteins and chromophore content being remarkably similar to that of LHCII [12]. It has been established that the red emission in these two LHCs originates from exciton/CT mixing in the Chl dimer 603-609 [45, 46, 84-86]. In LHCII, the distance between these two Chls is slightly larger, mainly due to their specific protein microenvironment. Specifically, in LHCII, Chl 603 is bound by the aminoacid histidine, while the pigment at the same site in PSI complexes is bound by an asparagine [2, 87].

SMS measurements on peripheral LHCs from PSI have demonstrated that these complexes are capable of reversibly switching off the CT character of the Chl 603-609 dimer (figure 5(b)) [83]. It was demonstrated that such behaviour is possible when the Chl-Chl distance is increased by as little as $0.74 \AA$ [88]. This suggests that only a subtle protein structural change is necessary to achieve such dramatic spectral tuning and hence also the capability of the complexes to absorb low-energy photons. The functional significance of this behaviour can be appreciated by considering that almost all visible light is absorbed by a single leaf, so that shade light contains an exceptionally high ratio of nearinfrared (i.e., $>700 \mathrm{~nm}$ ) photons. We conclude that the amount of shade light absorption by PSI and PSII is controlled by the specific protein microenvironment of the Chl 603-609 dimer through subtle structural changes brought about by the nature of a single amino acid. 


\section{Conclusion}

Although sounding paradoxical, we have explained that energy losses are important in photosynthetic light harvesting, the main function being to establish robustness. First, partial energy loss due to down-hill excitation energy transfer along energy gradients ensures a high quantum efficiency by minimizing complete energy loss (i.e., function-less trapping of an excitation). Second, photoprotection provides photosynthetic organisms with an amazing adaptability, and hence robustness, to large environmental changes.

The exceptionally high pigment densities of photosynthetic LHCs provide them with optimal absorption cross-sections. As a result, in most environments there is a surplus of absorbed light and the organisms safely dissipate all excess excitation energy in a fast and effective manner. Although energy losses are unavoidable for such high pigment concentrations, various benefits are provided by formation of molecular excitons to ensure ultrafast and efficient light harvesting. The indispensable energy losses are intelligently used by the organisms for photoprotection by establishing specific environments for the LHCs that control subtle and random protein conformational changes between light-harvesting and energy dissipating states. Such fine control establishes the probability of accessing different possible excitation traps in the complex. Carotenoids play an essential role in photoprotective energy dissipation, while charge-transfer states in pigment dimers also appear to be important.

Absorption of near-infrared light and subsequent uphill excitation energy transfer are used by various complexes to enhance light absorption in shade conditions or in environments where near-infrared light is prevalent. This capability is established by charge-transfer states in some Chl dimers, which can also be switched off by subtle protein conformational changes that move the Chls apart.

The light-harvesting apparatuses of photosynthetic organisms provide a powerful paradigm in which energy harvesting is carried out efficiently and robustly, despite their use of irregular, dynamic and 'defect-filled' proteins, in contrast to the best nano-engineered materials that are currently used in solar technologies.

\section{Acknowledgments}

The authors acknowledge past and present collaborators on this work: C. Ilioaia, R. Croce, E. Wientjes, J.M. Gruber, M.P. Johnson, A.V. Ruban, P. Horton, and V.I. Novoderezhkin. T.P.J.K. was supported by the University of Pretoria's Research Development Programme (Grant No. A0W679). R.v.G. was supported by an Advanced Investigator grant from the European Research Council (No. 267333, PHOTPROT), Nederlandse Organisatie voor Wetenschappelijk Onderzoek, Council of Chemical Sciences (NWO-CW) via a TOP-grant (700.58.305), and by the EU FP7 project PAPETS (GA 323901). Financial assistance of the National Research Foundation (NRF), South Africa, is gratefully acknowledged. Any opinion, findings and conclusions or recommendations expressed in this article are those of the authors, and therefore the NRF does not accept liability in regards thereto. 


\section{References}

[1] Kühlbrandt W, Wang D N and Fujiyoshi Y 1994 Atomic Model of Plant Light-Harvesting Complex by Electron Crystallography Nature 367 614-21

[2] Liu Z, Yan H, Wang K, Kuang T, Zhang J, Gui L, An X and Chang W 2004 Crystal structure of spinach major light-harvesting complex at 2.72 A resolution Nature 428 287-92

[3] Roduner E and Radhakrishnan S G 2016 In command of non-equilibrium Chem. Soc. Rev. 45 2768-84

[4] Novoderezhkin V I, Palacios M A, van Amerongen H and van Grondelle R 2005 Excitation dynamics in the LHCIl complex of higher plants: Modeling based on the $2.72 \AA$ crystal structure Journal of Physical Chemistry B 109 10493-504

[5] Demmig-Adams B and Adams W W 2002 Antioxidants in photosynthesis and human nutrition Science 298 2149-53

[6] Demmig-Adams B and Adams W W 2006 Photoprotection in an ecological context: the remarkable complexity of thermal energy dissipation New Phytol. 172 11-21

[7] Pascal A A, Liu Z F, Broess K, van Oort B, van Amerongen H, Wang C, Horton P, Robert B, Chang W R and Ruban A 2005 Molecular basis of photoprotection and control of photosynthetic light-harvesting Nature 436 134-7

[8] Caffarri S, Tibiletti T, Jennings R C and Santabarbara S 2014 A Comparison Between Plant Photosystem I and Photosystem II Architecture and Functioning Curr. Protein Pept. Sci. 15 296-331

[9] Van Amerongen H, Valkunas L and Van Grondelle R 2000 Photosynthetic excitons (Singapore: World Scientific Publishing )

[10] Scholes G D, Fleming G R, Olaya-Castro A and van Grondelle R 2011 Lessons from nature about solar light harvesting Nature Chemistry 3 763-74

[11] van Grondelle R and Novoderezhkin V I 2010 Quantum design for a light trap Nature 463 614-5

[12] Gobets B and van Grondelle R 2001 Energy transfer and trapping in photosystem I Biochim. Biophys. Acta - Bioenergetics 1507 80-99

[13] Bennett D I G, Amarnath K and Fleming G R 2013 A Structure-Based Model of Energy Transfer Reveals the Principles of Light Harvesting in Photosystem II Supercomplexes J. Am. Chem. Soc. 135 9164-73

[14] Pawlowicz N P, Groot M L, van Stokkum I H M, Breton J and van Grondelle R 2007 Charge separation and energy transfer in the photosystem II core complex studied by femtosecond midinfrared spectroscopy Biophysical Journal 93 2732-42

[15] Raszewski G and Renger T 2008 Light harvesting in photosystem II core complexes is limited by the transfer to the trap: Can the core complex turn into a photoprotective mode? J. Am. Chem. Soc. 130 4431-46

[16] van Grondelle R and Novoderezhkin V I 2006 Energy transfer in photosynthesis: experimental insights and quantitative models Phys. Chem. Chem. Phys. 8 793-807

[17] Beddard G S and Porter G 1976 Concentration quenching in chlorophyll Nature 260 366-7

[18] Kelly A R and Porter G 1970 Model Systems for Photosynthesis. I. Energy Transfer and Light Harvesting Mechanisms Proc R Soc London Ser A 324 117-26

[19] Beddard G S, Carlin S E and Porter G 1976 Concentration quenching of chlorophyll fluorescence in bilayer lipid vesicles and liposomes Chemical Physics Letters 43 27-32

[20] Katz J J, Shipman L L, Cotton T M and Janson T R 1978 The Porphyrins, ed C D Dolphin (New York: Academic Press) pp 401-58

[21] de Paula J C, Robblee J H and Pasternack R F 1995 Aggregation of chlorophyll-a probed by resonance light-scattering spectroscopy Biophysical Journal 68 335-41

[22] Helenius V M, Hynninen P H and Korppitommola J E I 1993 Chlorophyll a aggregates in hydrocarbon solution, a picosecond spectroscopy and molecular modeling study Photochemistry and Photobiology 58 867-73 
[23] Yuen M J, Shipman L L, Katz J J and Hindman J C 1980 Concentration quenching of fluorescence from chlorophyll-a, pheophytin-a, pyropheophytin-a and their covalently-linked pairs Photochemistry and Photobiology 32 281-96

[24] Knox R S 1994 Spectral effects of exciton splitting in statistical pairs J. Phys. Chem. 98 7270-3

[25] Seely G R 1978 Energetics of electron-transfer reactions of chlorophyll and other compounds Photochemistry and Photobiology 27 639-54

[26] Gutschick V P 1978 Concentration quenching in chlorophyll-alpha and relation to functional charge-transfer invivo J. Bioenerg. Biomembr. 10 153-70

[27] Kelly A R and Patterson L K 1971 Model systems for photosynthesis. 2. Concentration quenching of chlorophyll-b fluorescence in solid solutions Proceedings of the Royal Society of London Series a-Mathematical and Physical Sciences 324 117-+

[28] Parson W W and Warshel A 1987 Spectroscopic properties of photosynthetic reaction centers .2. Application of the theory to Rhodopseudomonas-viridis. J. Am. Chem. Soc. 109 6152-63

[29] Warshel A and Parson W W 1987 Spectroscopic properties of photosynthetic reaction centers.1. Theory J. Am. Chem. Soc. 109 6143-52

[30] Walters R G, Ruban A V and Horton P 1996 Identification of proton-active residues in a higher plant light-harvesting complex Proceedings of the National Academy of Sciences of the United States of America 93 14204-9

[31] Jahns P, Latowski D and Strzalka K 2009 Mechanism and regulation of the violaxanthin cycle: The role of antenna proteins and membrane lipids Biochimica Et Biophysica ActaBioenergetics 1787 3-14

[32] Li X P, Muller-Moule P, Gilmore A M and Niyogi K K 2002 PsbS-dependent enhancement of feedback de-excitation protects photosystem II from photoinhibition Proceedings of the National Academy of Sciences of the United States of America 99 15222-7

[33] Frank H A, Bautista J A, Josue J S and Young A J 2000 Mechanism of nonphotochemical quenching in green plants: Energies of the lowest excited singlet states of violaxanthin and zeaxanthin Biochemistry 39 2831-7

[34] Ruban A V, Berera R, Ilioaia C, van Stokkum I H M, Kennis J T M, Pascal A A, van Amerongen $H$, Robert B, Horton P and van Grondelle R 2007 Identification of a mechanism of photoprotective energy dissipation in higher plants Nature 450 575-8

[35] Miloslavina Y, Wehner A, Lambrev P H, Wientjes E, Reus M, Garab G, Croce R and Holzwarth A R 2008 Far-red fluorescence: A direct spectroscopic marker for LHCII oligomer formation in non-photochemical quenching Febs Letters 582 3625-31

[36] Muller M G, Lambrev P, Reus M, Wientjes E, Croce R and Holzwarth A R 2010 Singlet energy dissipation in the photosystem II light-harvesting complex does not involve energy transfer to carotenoids ChemPhysChem 11 1289-96

[37] Holt N E, Zigmantas D, Valkunas L, Li X P, Niyogi K K and Fleming G R 2005 Carotenoid cation formation and the regulation of photosynthetic light harvesting Science 307 433-6

[38] Zubik M, Luchowski R, Puzio M, Janik E, Bednarska J, Grudzinski W and Gruszecki W I 2013 The negative feedback molecular mechanism which regulates excitation level in the plant photosynthetic complex LHCII: Towards identification of the energy dissipative state Biochimica Et Biophysica Acta-Bioenergetics 1827 355-64

[39] Duffy C D P, Chmeliov J, Macernis M, Sulskus J, Valkunas L and Ruban A V 2013 Modeling of Fluorescence Quenching by Lutein in the Plant Light-Harvesting Complex LHCII Journal of Physical Chemistry B 117 10974-86

[40] Duffy C D P, Valkunas L and Ruban A V 2013 Quantum Mechanical Calculations of Xanthophyll-Chlorophyll Electronic Coupling in the Light-Harvesting Antenna of Photosystem II of Higher Plants Journal of Physical Chemistry B 117 7605-14

[41] Walla P J, Linden P A, Ohta K and Fleming G R 2002 Excited-state kinetics of the carotenoid S1 state in LHC II and two-photon excitation spectra of lutein and beta-carotene in solution: 
Efficient car S-1 -> Chl electronic energy transfer via hot S-1 states? J. Phys. Chem. A 106 1909-16

[42] van Amerongen $\mathrm{H}$ and van Grondelle R 2001 Understanding the energy transfer function of LHCII, the major light-harvesting complex of green plants J. Phys. Chem. B 105 604-17

[43] Liao P N, Holleboom C P, Wilk L, Kuhlbrandt W and Walla P J 2010 Correlation of Car S-1 -> Chl with Chl $\rightarrow$ Car S-1 Energy Transfer Supports the Excitonic Model in Quenched Light Harvesting Complex II J. Phys. Chem. B 114 15650-5

[44] Chmeliov J, Bricker W P, Lo C, Jouin E, Valkunas L, Ruban A V and Duffy C D P 2015 An 'all pigment' model of excitation quenching in LHCIl Phys. Chem. Chem. Phys. 17 15857-67

[45] Novoderezhkin V I, Croce R, Wahadoszamen M, Polukhina I, Romero E and van Grondelle R 2016 Mixing of exciton and charge-transfer states in light-harvesting complex Lhca4 Phys.

Chem. Chem. Phys. 18 19368-77

[46] Romero E, Mozzo M, van Stokkum I H M, Dekker J P, van Grondelle R and Croce R 2009 The Origin of the Low-Energy Form of Photosystem I Light-Harvesting Complex Lhca4: Mixing of the Lowest Exciton with a Charge-Transfer State Biophys. J. 96 L35-L7

[47] Croce R, Chojnicka A, Morosinotto T, Ihalainen J A, van Mourik F, Dekker J P, Bassi R and van Grondelle R 2007 The low-energy forms of photosystem I light-harvesting complexes: Spectroscopic properties and pigment-pigment interaction characteristics Biophys. J. 93 2418-28

[48] Reimers J R, Biczysko M, Bruce D, Coker D F, Frankcombe T J, Hashimoto H, Hauer J, Jankowiak R, Kramer T, Linnanto J, Mamedov F, Muh F, Ratsep M, Renger T, Styring S, Wan J, Wang Z A, Wang-Otomo Z Y, Weng Y X, Yang C H, Zhang J P, Freiberg A and Krausz E 2016 Challenges facing an understanding of the nature of low-energy excited states in photosynthesis Biochimica Et Biophysica Acta-Bioenergetics 1857 1627-40

[49] Vaitekonis $S$, Trinkunas $G$ and Valkunas $L 2005$ Red chlorophylls in the exciton model of photosystem I Photosynthesis Research 86 185-201

[50] Zazubovich V, Matsuzaki S, Johnson T W, Hayes J M, Chitnis P R and Small G J 2002 Red antenna states of photosystem I from cyanobacterium Synechococcus elongatus: a spectral hole burning study Chemical Physics 275 47-59

[51] Wahadoszamen M, Berera R, Ara A M, Romero E and van Grondelle R 2012 Identification of two emitting sites in the dissipative state of the major light harvesting antenna Phys. Chem. Chem. Phys. 14 759-66

[52] Chmeliov J, Gelzinis A, Songaila E, Augulis R, Duffy C D P, Ruban A V and Valkunas L 2016 The nature of self-regulation in photosynthetic light-harvesting antenna Nature Plants 2

[53] Krüger T P J, llioaia C, Johnson M P, Ruban A V, Papagiannakis E, Horton P and van Grondelle R 2012 Controlled Disorder in Plant Light-Harvesting Complex II Explains Its Photoprotective Role Biophys. J. 102 2669-76

[54] Johnson M P and Ruban A V 2009 Photoprotective Energy Dissipation in Higher Plants Involves Alteration of the Excited State Energy of the Emitting Chlorophyll(s) in the Light Harvesting Antenna II (LHCII) J. Biol. Chem. 284 23592-601

[55] Ruban A V and Horton P 1992 Mechanism of [Delta]pH-dependent dissipation of absorbed excitation energy by photosynthetic membranes. I. Spectroscopic analysis of isolated lightharvesting complexes Biochim. Biophys. Acta - Bioenergetics 1102 30-8

[56] Ruban A V, Rees D, Noctor G D, Young A and Horton P 1991 Long-wavelength chlorophyll species are associated with amplification of high-energy-state excitation quenching in higher-plants Biochimica Et Biophysica Acta 1059 355-60

[57] Ilioaia C, Johnson M P, Horton P and Ruban A V 2008 Induction of Efficient Energy Dissipation in the Isolated Light-harvesting Complex of Photosystem II in the Absence of Protein Aggregation J. Biol. Chem. 283 29505-12

[58] Johnson M P, Goral T K, Duffy C D P, Brain A P R, Mullineaux C W and Ruban A V 2011 Photoprotective Energy Dissipation Involves the Reorganization of Photosystem II Light- 
Harvesting Complexes in the Grana Membranes of Spinach Chloroplasts Plant Cell 23 146879

[59] Galland C, Ghosh Y, Steinbrueck A, Sykora M, Hollingsworth J A, Klimov V I and Htoon H 2011 Two types of luminescence blinking revealed by spectroelectrochemistry of single quantum dots Nature 479 203-U75

[60] Klimov V I, Mikhailovsky A A, McBranch D W, Leatherdale C A and Bawendi M G 2000 Quantization of multiparticle Auger rates in semiconductor quantum dots Science 287 10113

[61] Zondervan R, Kulzer F, Orlinskii S B and Orrit M 2003 Photoblinking of rhodamine 6G in poly(vinyl alcohol): Radical dark state formed through the triplet J. Phys. Chem. A 107 6770-6

[62] Amarie S, Standfuss J, Barros T, Kuhlbrandt W, Dreuw A and Wachtveitl J 2007 Carotenoid radical cations as a probe for the molecular mechanism of nonphotochemical quenching in oxygenic photosynthesis Journal of Physical Chemistry B 111 3481-7

[63] Gwizdala M G, Berera R, Kirilovsky D, Van Grondelle R and Krüger T P J 2016 Controlling light harvesting with light J. Am. Chem. Soc.,

[64] Krüger T P J, llioaia C, Johnson M P, Belgio E, Horton P, Ruban A V and Van Grondelle R 2013 The specificity of controlled protein disorder in the photoprotection of plants Biophys. J. 105 1018-26

[65] Chmeliov J, Valkunas L, Krueger T P J, llioaia C and van Grondelle R 2013 Fluorescence blinking of single major light-harvesting complexes New Journal of Physics 15

[66] Valkunas L, Chmeliov J, Kruger T P J, Ilioaia C and van Grondelle R 2012 How Photosynthetic Proteins Switch Journal of Physical Chemistry Letters 3 2779-84

[67] Maly P, Gruber J M, van Grondelle R and Mancal T 2016 Single Molecule Spectroscopy of Monomeric LHCII: Experiment and Theory Scientific Reports 6

[68] Liguori N, Periole X, Marrink S J and Croce R 2015 From light-harvesting to photoprotection: structural basis of the dynamic switch of the major antenna complex of plants (LHClI) Scientific Reports 5

[69] Santabarbara S, Horton P and Ruban A V 2009 Comparison of the Thermodynamic Landscapes of Unfolding and Formation of the Energy Dissipative State in the Isolated Light Harvesting Complex II Biophysical Journal 97 1188-97

[70] van Oort B, van Hoek A, Ruban A V and van Amerongen H 2007 Equilibrium between quenched and nonquenched conformations of the major plant light-harvesting complex studied with high-pressure time-resolved fluorescence Journal of Physical Chemistry B 111 7631-7

[71] Krüger T P J, llioaia C, Valkunas L and Van Grondelle R 2011 Fluorescence Intermittency from the Main Plant Light-Harvesting Complex: Sensitivity to the Local Environment J. Phys. Chem. B 115

[72] Rutkauskas D, Novoderezhkin V, Cogdell R J and van Grondelle R 2005 Fluorescence spectroscopy of conformational changes of single LH2 complexes Biophys. J. 88 422-35

[73] Schlau-Cohen G S, Wang Q, Southall J, Cogdell R J and Moerner W E 2013 Single-molecule spectroscopy reveals photosynthetic LH2 complexes switch between emissive states Proceedings of the National Academy of Sciences of the United States of America 11010899 903

[74] Krüger T P J, Ilioaia C and Van Grondelle R 2011 Fluorescence Intermittency from the Main Plant Light-Harvesting Complex: Resolving Shifts between Intensity Levels J. Phys. Chem. B 115

[75] Schlau-Cohen G S, Yang H-Y, Krueger T P J, Xu P, Gwizdala M, van Grondelle R, Croce R and Moerner W E 2015 Single-Molecule Identification of Quenched and Unquenched States of LHCII Journal of Physical Chemistry Letters 6 860-7

[76] Gruber J M, Chmeliov J, Kruger T P J, Valkunas L and van Grondelle R 2015 Singlet-triplet annihilation in single LHCll complexes Phys. Chem. Chem. Phys. 17 19844-53 
[77] Gruber J M, Xu P Q, Chmeliov J, Kruger T P J, Alexandre M T A, Valkunas L, Croce R and van Grondelle R 2016 Dynamic quenching in single photosystem II supercomplexes Phys. Chem. Chem. Phys. 18 25852-60

[78] van Oort B, van Grondelle R and van Stokkum I H M 2015 A Hidden State in Light-Harvesting Complex II Revealed By Multipulse Spectroscopy Journal of Physical Chemistry B 119 5184-93

[79] Ferretti M, Hendrikx R, Romero E, Southall J, Cogdell R J, Novoderezhkin V I, Scholes G D and van Grondelle R 2016 Dark States in the Light-Harvesting complex 2 Revealed by Twodimensional Electronic Spectroscopy Scientific Reports 6

[80] Wahadoszamen M, Margalit I, Ara A M, van Grondelle R and Noy D 2014 The role of chargetransfer states in energy transfer and dissipation within natural and artificial bacteriochlorophyll proteins Nature Communications 5

[81] Krüger T P J, Novoderezhkin V I, Ilioaia C and van Grondelle R 2010 Fluorescence Spectral Dynamics of Single LHCII Trimers Biophys. J. 98 3093-101

[82] Krüger T P J, Ilioaia C, Johnson M P, Ruban A V and van Grondelle R 2014 Disentangling the low-energy states of the major light-harvesting complex of plants and their role in photoprotection Biochimica Et Biophysica Acta-Bioenergetics 1837 1027-38

[83] Krüger T P J, Wientjes E, Croce R and van Grondelle R 2011 Conformational switching explains the intrinsic multifunctionality of plant light-harvesting complexes Proc Natl Acad Sci USA 108 13516-21

[84] Morosinotto T, Breton J, Bassi R and Croce R 2003 The nature of a chlorophyll ligand in Lhca proteins determines the far red fluorescence emission typical of photosystem I Journal of Biological Chemistry 278 49223-9

[85] Morosinotto T, Mozzo M, Bassi R and Croce R 2005 Pigment-pigment interactions in Lhca4 antenna complex of higher plants photosystem I Journal of Biological Chemistry 280 20612-9

[86] Mozzo M, Morosinotto T, Bassi R and Croce R 2006 Probing the structure of Lhca3 by mutation analysis Biochimica Et Biophysica Acta-Bioenergetics 1757 1607-13

[87] Ben-Shem A, Frolow F and Nelson N 2003 Crystal structure of plant photosystem I Nature 426 630-5

[88] Wientjes E, Roest G and Croce R 2012 From red to blue to far-red in Lhca4: How does the protein modulate the spectral properties of the pigments? Biochimica Et Biophysica ActaBioenergetics 1817 711-7 